\title{
Therapeutic hypothermia and myocardium in perinatal asphyxia: a microvolt T-wave alternans and Doppler echocardiography study
}

\author{
Karpuz D ${ }^{1}$, Celik $\mathrm{Y}^{2}$, Giray $\mathrm{D}^{1}$, Tasdelen $\mathrm{B}^{3}$, Hallioglu $\mathrm{O}^{1}$ \\ Department of Pediatrics, Division of Pediatric Cardiology, University of Mersin Faculty of Medicine, \\ Mersin, Turkey.drderyakarpuz@gmail.com
}

\section{ABSTRACT}

OBJECTIVES: This is the first study evaluating the predictive value of myocardial performance on arrhythmia and mortality via tissue-Doppler and microvolt T-wave alternans in infants with hypoxic-ischemic encephalopathy treated with therapeutic hypothermia-rewarming.

METHODS: The study included 23 term newborns having criteria for hypoxic-ischemic encephalopathy, and 12 controls. Tissue-Doppler imaging and T-wave alternans were performed in the first six hours after birth in patients from both groups and after hypothermia-rewarming treatment on the fifth day.

RESULTS: The basal T-wave alternans values were higher in patients in lead aVF $(p<0.001)$ which also correlated with existing acidemia $(r=0.517 ; p=0.012)$. Basal T-wave alternans and post-treatment values of patients were compared in leads V1 $(p<0.001)$ and aVF $(p<0.001)$; a significant decrease was found on the fifth day. Moreover, right ventricle diastolic diameter and estimated systolic pulmonary artery pressure of patients in the first 6 hours were higher $(p=0.03, p<0.001$, respectively). Although, the ejection fraction of patients did not decrease, basal values of left and right ventricular systolic and diastolic functions were lower initially, and increased significantly after treatment.

CONCLUSION: The global cardiac functions and myocardial performance of newborns with hypoxic-ischemia might be improved with therapeutic hypothermia which can be determined by using T-wave alternans and tissueDoppler measurements. However, further studies are needed to assess whether these measurements are prognostic in determining the myocardial dysfunction and arrhythmias (Tab. 2, Fig. 3, Ref. 26). Text in PDF www.elis.sk. KEYWORDS: hypoxic-ischemic encephalopathy, microvolt T-wave alternans, therapeutic hypothermia, tissue Doppler.

\section{Introduction}

Hypoxic-ischemic encephalopathy is the major cause of mortality and morbidity in neonatal period $(1,2)$. The mortality rate was reported as $10-60 \%$ in infants with hypoxic-ischemic encephalopathy which is an important cause of admission to neonatal intensive care units with multi-organ dysfunction and presence of brain, heart and renal injury is significantly associated with poor prognosis $(1,3)$ Hypoxia causes damage of the papillary muscles and myocardial tissue by afflicting cellular injury to the myocardium (4) The resulting myocardial dysfunction is one of the important parameters determining the prognosis (3). The incidence of cardiac dysfunction in perinatal asphyxia is in range of 24-60 $\%$ (5). The tissue Doppler imaging is known being better than conventional echocardiography in evaluating the systolic and diastolic functions of the ventricles. Microwolt T-wave alternans

${ }^{1}$ Department of Pediatrics, Division of Pediatric Cardiology, University of Mersin Faculty of Medicine, Mersin, Turkey, ${ }^{2}$ Department of Pediatrics, Division of Neonatology, University of Mersin Faculty of Medicine, Mersin, Turkey, and ${ }^{3}$ Department of Biostatistics, University of Mersin Faculty of Medicine, Mersin, Turkey

Address for correspondence: D. Karpuz, MD, Department of Pediatrics, Divison of Pediatric Cardiology, University of Mersin Faculty of Medicine 34. Cadde, Ciftlikkoy Kampusu 33343 Mersin, Turkey.

Phone: +90.324.241.0000, Fax: +90.324.241.0000 is a well-known noninvasive predictor of ventricular arrhythmias in patients with cardiomyopathies $(6,7)$. Although there is some limited number of studies related to microwolt T-wave alternans in adult patients, there is not any study on newborns with hypoxic-ischemic encephalopathy. It has been reported that the rate of mortality and neurodevelopmental morbidity decrease with therapeutic hypothermia in infants with moderate and severe hypoxic-ischemic encephalopathy $(8,9)$. However, there is no study investigating the effect of therapeutic hypothermia on cardiac dysfunction. This is the first study aiming to evaluate the predictive value of myocardial performance on arrhythmia and mortality with the use of tissue Doppler imaging and microwolt T-wave alternans in hypoxic infants who were endangered with therapeutic hypothermia and rewarming.

\section{Methods}

\section{Study group}

This prospective randomized study was done at the newborn intensive care unit during a period from July 2016 to November 2016. The diagnosis of hypoxic-ischemic encephalopathy was based on American College of Obstetricians and Gynecologists criteria (10). The severity of hypoxic-ischemic encephalopathy was determined according to modified Sarnat staging (11). This study included 23 term newborns having the diagnostic criteria 
for hypoxic-ischemic encephalopathy and scored using Sarnat staging as well as age and gender-matched 12 healthy infants as control. The exclusion criteria were arriving at the center $>6 \mathrm{~h}$ after birth, having gestational age $<36$ weeks, having severe intrauterine growth retardation, congenital heart disease or severe congenital anomalies. Patients who meet the inclusion criteria and who were diagnosed with hypoxic-ischemic encephalopathy, having $10 \mathrm{~min}$ Apgar score $\leq 5$, those who received positive pressure ventilation until $10 \mathrm{~min}$ after delivery, or those with $\mathrm{pH}<7.0$, or base excess $\leq-16$ based on blood gas analysis within the first hour after birth were evaluated further. Amplitude-integrated electroencephalography (aEEG) records (Brainz; Natus Medical, San Carlos, CA, USA) were kept for at least $30 \mathrm{~min}$ to consider any finding(s) indicative of moderate or severe encephalopathy (i.e. lethargy, stupor, and coma), hypotonia, abnormal reflexes (i.e. pupil response to light), or convulsion. Only patients with moderate and severe hypoxic-ischemic encephalopathy were included in the study. Infants were cooled using the manually controlled cool cap (Olympic Medical Cool Care System; Olympic Medical, Seattle, WA, USA) for 72 hours which was followed by rewarming over 6 hours. In the hypoxic-ischemic encephalopathy group, the aim was to maintain rectal temperature at $33-34{ }^{\circ} \mathrm{C}$ for $72 \mathrm{~h}$. After 72 $\mathrm{h}$ of cooling, the rectal temperature was increased to $36.5^{\circ} \mathrm{C}$ at $\leq$ $0.5^{\circ} \mathrm{C} / \mathrm{h}$. Patients who were not in the target temperature range for $>1 \mathrm{~h}$ were excluded. Myocardial involvement was assessed with pulse and tissue Doppler echocardiography and microwolt T-wave alternans which were performed in the first six hours after birth, and after hypothermia-rewarming treatment on the fifth day of life.

The control group consisted of healthy individuals with normal echocardiographic and electrocardiographic examinations, and without history of cardiovascular disease. Consents of the local ethics committee were taken (2016/204).

\section{Echocardiography}

Both the patient and control groups were evaluated by transthoracic echocardiography (Vivid S5 Pro Ultrasound System (GE Medical Systems, Horten, Norway)) by using $6 \mathrm{MHz}$ transducers. All subjects underwent a complete 2-dimensional spectral Doppler, and color flow Doppler examinations. Echocardiographic examinations were performed by the same experienced echocardiographer. Three cardiac cycles were recorded to reduce the influence of respiration on myocardial velocities. The left ventricle ejection fraction was calculated by using Simpson's biplane method. Right ventricle morphology was defined by using right ventricular end-diastolic diameter. Tricuspid regurgitation jet and ventricular septal flattening were used to assess pulmonary hypertension in conventional echocardiography. Tissue Doppler velocities were obtained at the apical 4-chamber view. Sample volume was placed at the septal and lateral ages of the annulus of mitral and tricuspid valves, and at medium and apical areas of left ventricle, interventricular septum and right ventricle. It was important to minimize the incidence angle between the Doppler beam and the direction of the myocardial wall motion. Doppler signal quality was enhanced by lowering the Nyquist limit to 10 to $30 \mathrm{~cm} / \mathrm{s}$, using the lowest wall filter settings with minimal optimal gain, decreasing Doppler sample volume size to $5 \mathrm{~mm}$, and optimizing the sweep speed at least to $100 \mathrm{~mm} / \mathrm{s}$. Peak early diastolic (Em), peak atrial systolic (Am), and peak systolic (Sm) myocardial velocities were recorded. At the same time, Em / Am ratio was calculated.

\section{Holter ECG and microvolt T-wave alternans measurement}

A 24-hour Holter electrocardiography (ECG) monitoring was done by use of a digital recording device (Seer MC) produced by GE Medical Systems, Nogoya, Japan in both groups. The measurements were taken from 3 derivations including V5, V1 and AVF. A sampling velocity of $128 \mathrm{~Hz}$ was selected. In both groups, the recordings were obtained in sinus rhythm. Microwolt T-wave alternans analysis was performed in form of maximum microwolt T-wave alternans analysis by way of the 7.2 version of the software produced by Mars PC systems. The method used for analysis was time domain Modified Moving Average. Microwolt T-wave alternans was analyzed from routine 24-hour Holter ECG recording based on V5, V1 and AVF derivations.

\section{Statistical analysis}

The data were processed and analyzed by using the STATA MP/11 statistical package. Normality assumption for continuous echocardiographic parameters and lead values was checked by Shapiro Wilk test. These variables were summarized as mean and standard deviations and the comparisons between patient and healthy control groups were performed using independent t-test

Tab. 1. Baseline characteristics of patient and control groups.

\begin{tabular}{|c|c|c|c|}
\hline & Patient $(n=23)$ & Control $(n=12)$ & $\mathrm{p}$ \\
\hline Gestational age (weeks) & $39.09 \pm 1.35$ & $38.58 \pm 1.08$ & 0.271 \\
\hline Birth weight $(\mathrm{g})$ & $3391.52 \pm 413.69$ & $3399.17 \pm 84.58$ & 0.950 \\
\hline Birth height $(\mathrm{cm})$ & $50.61 \pm 1.44$ & $50.08 \pm 1.68$ & 0.339 \\
\hline Head circumference $(\mathrm{cm})$ & $35.52 \pm 0.99$ & $35.08 \pm 0.79$ & 0.195 \\
\hline $\begin{array}{l}\text { Delivery } \\
\text { Vaginal delivery } \\
\text { Cesarean section } \\
\end{array}$ & $\begin{array}{c}9(39.1 \%) \\
14(60.9 \%)\end{array}$ & $\begin{array}{l}5(41.7 \%) \\
7(58.3 \%)\end{array}$ & 0.582 \\
\hline $\begin{array}{l}\text { Gender } \\
\text { Male } \\
\text { Female } \\
\end{array}$ & $\begin{array}{c}17(73.9 \%) \\
6(26.1 \%)\end{array}$ & $\begin{array}{l}5(41.7 \%) \\
7(58.3 \%)\end{array}$ & 0.079 \\
\hline Clinical convulsions & $11(47.8 \%)$ & - & $<0.001$ \\
\hline $\begin{array}{l}5 \text { min Apgar score } \\
0-3 \\
4-6 \\
7-10 \\
\end{array}$ & $\begin{array}{c}6(26.1 \%) \\
17(73.9 \%) \\
0\end{array}$ & $\begin{array}{c}- \\
- \\
12(100 \%)\end{array}$ & \\
\hline $\begin{array}{l}10 \text { min Apgar score } \\
0-3 \\
4-6 \\
7-10 \\
\end{array}$ & $\begin{array}{c}4(17.3 \%) \\
19(82.6) \\
0\end{array}$ & $\begin{array}{c}- \\
- \\
12(100 \%)\end{array}$ & \\
\hline $\begin{array}{l}\text { Blood gases within } 1 \mathrm{~h} \text { of birth } \\
\mathrm{pH} \leq 7.00 \\
\text { Base excess }(\mathrm{mmol} / \mathrm{L})\end{array}$ & $\begin{array}{c}13(56.3 \%) \\
-13 \pm 3.5\end{array}$ & $\begin{array}{l}- \\
-\end{array}$ & \\
\hline $\begin{array}{l}\text { aEEG } \\
\text { Moderately abnormal } \\
\text { Severely abnormal }\end{array}$ & $\begin{array}{c}15(65.2 \%) \\
8(34.8 \%)\end{array}$ & $\begin{array}{c}12(100 \%) \\
-\end{array}$ & \\
\hline $\begin{array}{l}\text { Sarnat stage } \\
\text { II } \\
\text { III }\end{array}$ & $\begin{array}{l}15(65.2 \%) \\
8(34.8 \%)\end{array}$ & $\begin{array}{l}- \\
-\end{array}$ & \\
\hline
\end{tabular}



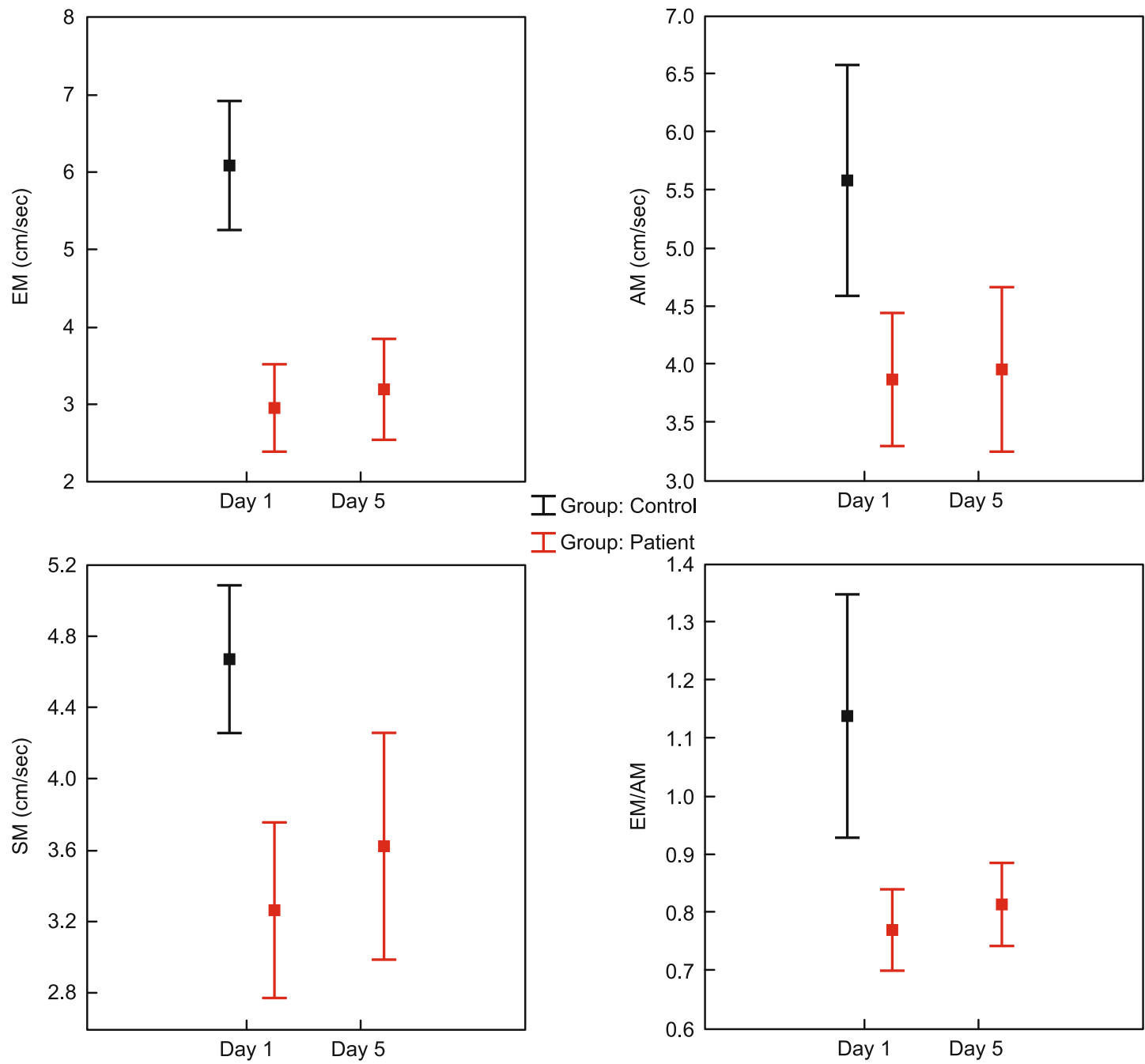

Fig. 1. Mean velocities of EM, AM, SM and EM/AM waves in tissue Doppler imaging of left ventricle of the patient and control and the difference between days 1 and 5 of the patients are shown.

or Mann-Whitney $U$ test. The change in lead values between the first and fifth days were analyzed using Paired t-test. The comparisons for categorical variables were done using chi-square test or Fisher's exact test. The correlations between microwolt T-wave alternans lead aVF values and the others were also investigated using Pearson correlation coefficient. Statistical significance level was assessed as less than 0.05 .

\section{Results}

During the period of study, 29 newborns were diagnosed as suffering from hypoxic-ischemic encephalopathy. Of them, 6 were excluded for not fulfilling the inclusion criteria. Two were excluded being aged $>6 \mathrm{~h}$ at the time of randomization, one had congenital malformation, one was excluded because of inability to evaluate the ECG records and two were excluded because they were hemodynamically unstable, required recurrent resuscitation and soon died. Thus, hypoxic-ischemic encephalopathy group included 23 patients and control group included 12 newborns. Mean gestational age, gender, birth weight, head circumference, type of delivery did not differ in between the groups. Apgar score of the hypoxic infants was $4.43 \pm 0.99$ at 5 th minute. Mean $\mathrm{pH}$ value of the arterial blood gas was $6.85 \pm 0.23$ and base deficit was $13.43 \pm 3.56$ within the first hour of life. Twenty infants $(87 \%)$ were in Sarnat 2 and only $3(13 \%)$ were in Sarnat 3. In aEEG evaluation, 15 patients $(65.2$ $\%$ ) had moderate and 8 patients (34.8\%) had severe impairment (Tab. 1). Newborns with hypoxic-ischemic encephalopathy were evaluated in the first six hours after birth and on fifth day of life for hypotension, bradycardia, thrombocytopenia, abnormal coagulation test, abnormal renal function, electrolyte imbalance, elevation of liver enzymes, sepsis with culture positivity, persistent pulmonary hypertension, pulmonary air leak, need for mechanical ventilation or extubation during the cooling period, but there were no significant differences between any of them. None of the patients 
Tab. 2. Echocardiographic parameters between the groups.

\begin{tabular}{|c|c|c|c|c|c|}
\hline & \multirow[t]{2}{*}{ Control } & \multicolumn{4}{|c|}{ Patient } \\
\hline & & 1st day & 5 th day & p value ${ }^{1}$ & $\mathrm{p}$ value $^{2}$ \\
\hline LVEDD & $14.17 \pm 3.16$ & $15.83 \pm 2.74$ & $16.38 \pm 3.15$ & 0.116 & 0.540 \\
\hline LVESD & $7.58 \pm 2.47$ & $8.96 \pm 2.50$ & $9.76 \pm 1.72$ & 0.130 & 0.360 \\
\hline RVDD & $15.81 \pm 1.60$ & $17.61 \pm 2.49$ & $17.32 \pm 2.59$ & 0.030 & 0.601 \\
\hline$\overline{R V D D / L V E D D ~}$ & $1.17 \pm 0.34$ & $1.13 \pm 0.19$ & $1.07 \pm 0.38$ & 0.617 & 0.551 \\
\hline $\mathrm{LA} / \mathrm{AO}$ & $0.99 \pm 0.37$ & $1.12 \pm 0.35$ & $1.05 \pm 0.35$ & 0.312 & 0.299 \\
\hline EF & $78.67 \pm 10.47$ & $73.48 \pm 10.03$ & $76.57 \pm 10.09$ & 0.162 & 0.341 \\
\hline sPAPecho & $20.78 \pm 8.08$ & $38.59 \pm 16.92$ & $25.56 \pm 10.01$ & $<0.001$ & 0.010 \\
\hline dPAPecho & $11.18 \pm 1.62$ & $18.55 \pm 8.14$ & $14.18 \pm 3.22$ & $<0.001$ & 0.045 \\
\hline Mitral E/A & $1.04 \pm 0.27$ & $1.08 \pm 0.42$ & $1.14 \pm 0.38$ & 0.780 & 0.580 \\
\hline Mitral MPI & $0.54 \pm 0.17$ & $0.58 \pm 0.26$ & $0.57 \pm 0.15$ & 0.931 & 0.113 \\
\hline Tricuspid E/A & $0.79 \pm 0.25$ & $0.87 \pm 0.34$ & $0.78 \pm 0.20$ & 0.468 & 0.329 \\
\hline Tricuspid MPI & $0.40 \pm 0.15$ & $0.38 \pm 0.21$ & $0.36 \pm 0.21$ & 0.774 & 0.884 \\
\hline
\end{tabular}

A - Late ventricular filling velocities; AO - Aorta; E - Early ventricular filling velocities EF - Ejection fraction; LA - Left atrium; LVEDD - Left ventricular end-diastolic diameter; LVESD - Left ventricular end- systolic diameter; MPI - Myocardial performance index; RVEDD - Right ventricular end-diastolic diameter; sPAPecho - Systolic pulmonary artery pressure (echocardiography); dPAPecho - Diastolic pulmonary artery pressure (echocardiography). $p$ value ${ }^{1}$ : For the comparison between control and patient groups on 1st day. $\mathrm{p}$ value ${ }^{2}$ : For the comparison between 1 st and 5 th days of patients
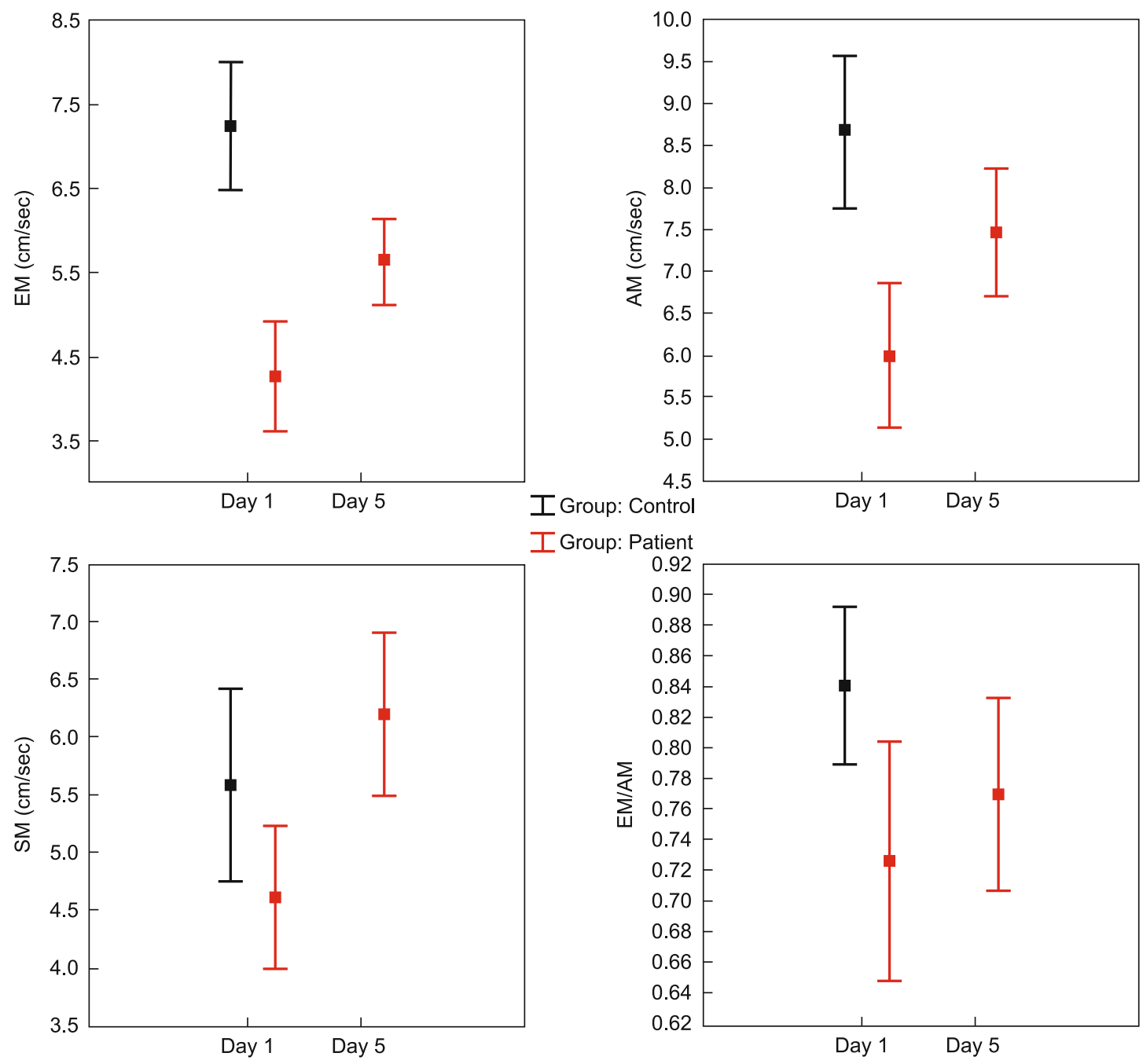

Fig. 2. Mean velocities of EM, AM, SM and EM/AM waves in tissue Doppler imaging of right ventricle of the patient and control and the difference between days 1 and 5 of the patients are shown. 
had hypoglycemia, necrotizing enterocolitis, or high hemoconcentration. Dopamine was used as a positive inotropic agent during the ventilation therapy of these patients. Tachycardia or bradycardia episodes were not observed in the patients during the study.

Mean velocities of Em, Am and Sm waves in the left ventricle and right ventricle were significantly lower in hypoxic group on the first day of life $(\mathrm{p}<0.05)$. Also, Em / Am values in transmitral tissue Doppler imaging were significantly lower than in the control group on the first day $(\mathrm{p}=0.001)$ (Figs 1 and 2, respectively). Myocardial performance index values of both ventricles measured with Pulse Doppler on the first day of life were higher in patients, but were not statistically significant. In the hypoxic group, right ventricular end-diastolic diameter, estimated systolic and diastolic pulmonary arterial pressures calculated from the tricuspid regurgitation and pulmonary regurgitation values on the first day of life were significantly higher $(\mathrm{p}=0.03, \mathrm{p}=0.001, \mathrm{p}=0.002$, respectively) (Tab. 2). In patients after cooling and rewarming treatment on the fifth day of life, mean velocities of Em, Am and Sm waves in the left ventricle and right ventricle were significantly higher than those recorded on the first day of life $(p<0.05)$. There was no significant change in transmitral tissue Doppler analysis of Em / Am values between those measured on the first and fifth days (Figs 1 and 2, respectively). Myocardial performance index values measured with Pulse Doppler were slightly decreased after cooling treatment on the fifth day, but were not significant. Hypoxic infants had a decrease in tricuspid regurgitation and pulmonary regurgitation values on fifth day when compared with those on the first day of life, but this decrease was significant only in pul-

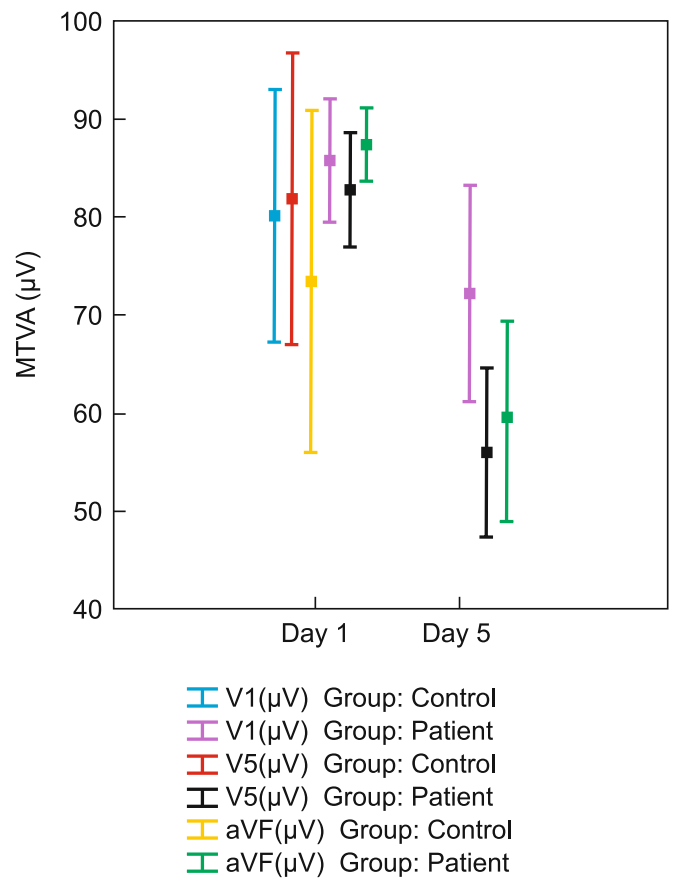

Fig. 3. Microwolt T-wave alternans values of the patient and control and the difference between days 1 and 5 of the patients are shown. (MTWA = Microwolt T-wave alternans values) monary regurgitation value $(\mathrm{p}=0.049)$. Other echocardiographic parameters are showed in Table 2.

Microwolt T-wave alternans values; lead V5 was $85.74 \pm 14.47$ $\mu \mathrm{V}$ in the patient group $(80.08 \pm 20.24 \mu \mathrm{V}$ in the control group), lead $\mathrm{V} 1$ was $82.74 \pm 13.71 \mu \mathrm{V}(81.83 \pm 23.32 \mu \mathrm{V}$ in the control group), and lead aVF was $87.39 \pm 8.63 \mu \mathrm{V}(73.50 \pm 27.43 \mu \mathrm{V}$ in the control group). The microwolt T-wave alternans values were compared; only lead aVF values were statistically higher in patients $(\mathrm{p}<$ 0.001 ) (Figure 3). Microwolt T-wave alternans values of patients on the first day of life in lead V5 were $85.74 \pm 14.47 \mu \mathrm{V}(72.14 \pm 24.03$ $\mu \mathrm{V}$ on the fifth day of life), in lead $\mathrm{V} 1$ they were $82.74 \pm 13.71 \mu \mathrm{V}$ ( $56.05 \pm 18.95 \mu \mathrm{V}$ on the fifth day of life), and in lead aVF they were $87.39 \pm 8.63 \mu \mathrm{V}(59.24 \pm 22.48 \mu \mathrm{V}$ on the fifth day of life). Baseline microwolt T-wave alternans values were compared with the values after hypothermia and rewarming, and values of lead V1 and aVF decreased significantly after treatment $(p<0.001, p<0.001)$ (Fig. 3).

In infants with hypoxic-ischemic encephalopathy microwolt $\mathrm{T}$ wave alternans values in lead aVF on first day of life were significantly correlated with the severity of acidosis $(r=0.517 \mathrm{p}=0.012)$. There was no significant relationship between microwolt T-wave alternans values and aEEG findings and hospitalization period.

\section{Discussion}

This is the first study evaluating the cardiac effects of therapeutic hypothermia and rewarming with microwolt T-wave alternans and tissue Doppler imaging. It has been determined that the microwolt T-wave alternans values were higher in infants with hypoxic-ischemic encephalopathy when compared to healthy newborns, and reduced significantly with the treatment of therapeutic hypothermia. Moreover, it was shown that in patients with hypoxic-ischemic encephalopathy, microwolt T-wave alternans values in lead aVF, which were significantly higher, were significantly correlated with the severity of acidosis. Furthermore, by tissue Doppler imaging it was shown that decreased tissue systolic and diastolic functions significantly improved with the therapeutic hypothermia on the fifth day.

The severity and duration of perinatal asphyxia determines the degree of post-asphyxia cardiac dysfunction. Van Bel et al. showed left ventricular dysfunction especially on the first two days in infants with asphyxia (12). The left ventricular dysfunction may result in end-organ hypoperfusion and lead to aggravation of hypoxic/acidotic condition. Due to clinical utility of clinical signs, ECG and echocardiographic changes remain unknown; diagnosis of myocardial dysfunction is not easy. Myocardial performance index and tissue Doppler techniques, which are being used increasingly, are effective and noninvasive methods to evaluate hemodynamic impairment and global systolic and diastolic functions (13). In this study, echocardiographic data were evaluated on the first five days of life. It has been known that the effect of perinatal asphyxia on heart on the first days of life is more distinctive. Moreover, very few studies with healthy newborns have demonstrated that major myocardial circulatory changes appeared to be particularly on the first day whereas with little or no changes on the following days (14). In our study, the mean velocities of Em, Sm, and left 
ventricle and ratio of transmitral Em / Am on the first day were statistically lower in hypoxic group, similar to the data in literature. Although, the myocardial performance index values measured with Pulse Doppler in both ventricles were higher in the asphyxic group, it was not significant. Wei et al. showed that tissue Doppler imaging Sm values were lower in asphyxic group. They also showed that tissue Doppler imaging Sm was more sensitive than M mode echocardiography in evaluating left ventricular systolic function in newborns with asphyxia (15). In another study, tissue Doppler imaging deformation indices of myocardial function in the asphyxic group were found lower than that of the control on the first day of life (16). Despite limited numbers of studies evaluating the cardiac functions with post therapeutic hypothermia-rewarming processes, results seem to be confusing. Zhou et al. did not find significant differences in cardiac functions between normothermic asphyxiated newborns and asphyxiated newborns treated with hypothermia (17). In another study including seven asphyxiated patients, it was shown that cardiac output was decreased as to 67 $\%$ of normal with whole-body hypothermia and passive rewarming (18). In two recent studies, Sehgal et al had found that global cardiac functions were low in infants undergoing hypothermia (19). Nestaas, et al. had showed an improvement in deformation indices with the tissue Doppler imaging on myocardial function when compared to the first day in the cooled group after rewarming (16). In our study, the echocardiographic evaluation after the therapeutic hypothermia and rewarming revealed that the tissue Doppler imaging parameters of both ventricles were significantly higher than those recorded on the first day of life. Moreover, myocardial performance index values evaluated with the pulse Doppler had an improvement after cooling treatment on fifth day, but were not statistically significant. Nestaas at al. showed such a similar improvement in asphyxiated infants in a serial echocardiographic evaluation on first day of life and post-treatment on fourth day also (16). Sehgal et al. performed echocardiography to the asphyxiated infants treated with hypothermia only on the first day of life. Since, this study was not a serial echocardiography evaluation, it is difficult to determine the effect of 72 hours of cooling and rewarming therapy on cardiac functions. Neonatal heart works at maximal of contractility (16). The improvement in cardiac function of asphyxiated infants with therapeutic hypothermia and rewarming can be explained by the preserved ability of the heart to maintain working at a high level of contractility. In addition, tissue Doppler imaging may provide earlier diagnostic values in assessing cardiac function of newborns with the history of asphyxia in the follow-up.

The influence of hypoxemia is related to myocardial dysfunction in asphyxiated neonates and is thought to be secondary to direct effect of ischemia of cardiac myocytes (20). Thus, changes in ECG may be seen as a result of myocardial hypoxia in asphyxiated newborns. Microwolt T-wave alternans is an electrocardiographic phenomenon displaying inhomogeneity of the myocardial repolarization process. The inhomogeneity of the intracardiac repolarization leads to a dispersion of the depolarizing wave, reentry phenomenon and, resulting in initiation of the ventricular arrhythmia. Prognostic value of microwolt T-wave alternans was proven in ischemic heart diseases, heart failure and cardiomyopa- thies (21-23). On the other hand, there was not any study using microwolt T-wave alternans measurements for asphyxic infants in literature. This is the first study measuring microwolt T-wave alternans with time-domain Modified Moving Average method in asphyxiated infants with therapeutic hypothermia and rewarming. In children with congenital and/or acquired heart diseases, there were only two studies using the time-domain Modified Moving Average method microwolt T-wave alternans. In this study, Makarov et al. compared 63 healthy children with 85 pediatric patients with congenital or acquired cardiovascular disease and microwolt T-wave alternans values were found significantly higher in patient group (24). In another study, Makarov et al. were evaluated 20 healthy newborns on first, second and fourth days after birth with the same technique, and the average peak value of T-wave alternans was found to be $32 \pm 8(12-55) \mu \mathrm{V}$ (25). In our study, microwolt T-wave alternans values in all leads were higher in asphyxiated infants than in controls, suggesting cardiac impairment in asphyxiated infants. In our study, there was also a control group in which microwolt T-wave alternans was done within the first 24 hours. However, the microwolt T-wave alternans values in the control group were higher than those of Makarov et al. The high microwolt T-wave alternans values in the control group in our study can be explained by the fact that microwolt T-wave alternans was done in the first 24 hours, and especially on the first day, the major changes may occur in myocardial circulation in neonates.

It has been shown that hypothermia may protect cardiac autonomic regulation in critically ill patients and also that it is associated with favorable results in heart rate variability in patients treated with hypothermia (26). In our study, significantly reduced initial microwolt T-wave alternans values with hypothermia and rewarming therapy and improvement of tissue Doppler measurements support the efficacy of treatment. No serious arrhythmia was observed in Holter ECG recordings of our patients, because hemodynamically unstable patients were not included in the study and there was no death in the patient group during the study. In addition, the high microwolt $\mathrm{T}$-wave alternans values were correlated with the severity of acidosis, but not with patients' day of hospitalization and aEEG values. However, the prognostic effect of microwolt T-wave alternans cannot be fully explained without assessing the long-term neurofunctional development of these patients.

Microwolt T-wave alternans has been a widely used method, studied retrospectively and prospectively aiming to better identify patients at increased risk of sudden cardiac death (25). However, there are very few data about peculiarities of microwolt T-wave alternans in children, or even non-existent in asphyxic babies. In order to determine the cut-off levels of microwolt T-wave alternans as well as their possible predictable values for arrhythmia or cardiovascular mortality in these patients, further studies are required. Although in other studies, microwolt T-wave alternans values have been shown to be effective in determining the development of arrhythmia, the prognostic value of microwolt T-wave alternans has not been clearly demonstrated in our study since arrhythmia and sudden death did not occur in the patient group. For definitive outcomes, there is a need of long-term follow-up studies to determine the risk of sudden death and arrhythmia. 
There are some limitations to the current study. The absence of the control group on the fifth day is a limitation to this study, since the hemodynamics of the heart has a rapid change in the neonatal period. The association between hypoxic-ischemic insults and increased microwolt T-wave alternans may be related to several factors such as the direct effects of hypoxia on the myocardium, brainstem hypoxia leading to autonomic dysfunction, impact of inotropic drugs or effects of other critical care interventions. There were no significant differences in biochemical or hematological parameters and treatments that were applied during the cooling period in patients with hypoxic ischemic encephalopathy between the first six hours after birth and fifth day of life in our study.

In conclusion, the global cardiac functions of newborns with hypoxic-ischemic encephalopathy are negatively related to the severity of asphyxia. We conclude that the myocardial dysfunction and arrhythmia predisposition might be improved with therapeutic hypothermia which can be determined by using microwolt T-wave alternans and tissue Doppler measurements. However, further studies are needed to assess whether these measurements are prognostic in determiningmyocardial dysfunction and arrhythmias in newborns.

\section{References}

1. Jacobs SE, Berg M, Hunt R, Tarnow-Mordi WO, Inder TE, Davis PG. Cooling for newborns with hypoxic ischaemic encephalopathy. Cochrane Database Syst Rev 2013; (1): CD003311. doi: 10.1002/ 14651858.

2. Takenouchi T, Iwata $\mathrm{O}$, Nabetani M, Tamura M. Therapeutic hypothermia for neonatal encephalopathy. Brain Dev 2012; 34: 165-170.

3. Shah P, Riphagen S, Beyene J, Perlman M. Multiorgan dysfunction in infants with post-asphyxial hypoxic-ischaemic encephalopathy. Arch Dis Child Fetal Neonatal Ed 2004; 89: 152-155.

4. Levene MI, de Vries L. Hypoxic-ischemic encephalopathy. 938-956. In: Martin FJ, Fanaroff AA, Walsh MC (Eds). In Fanaroff and Martin's NeonatalPerinatal Medicine. Disease of the Fetus and Infant, 8th edn. Philadelphia: Mosby Elsevier, 2006.

5. Adcock LM, Papile LA. Perinatal asphyxia. 518-523. In: Cloherty JP, Eichenwald EC (Eds). Stark AR Manual of neonatal care. New Delhi: Wolters Kluwer; 2008.

6. Chow T, Kereiakes DJ, Bartone C et al. Prognostic utility of microvolt T-wave alternans in risk stratification of patients with ischemic cardiomyopathy. J Am Coll Cardiol 2006; 47 (9): 1820-1827.

7. Gupta A, Hoang D, Karliner L, Tice JA, Heidenreich P, Wang PJ, Turakhia MP. Ability of microvolt T-wave alternans to modify risk assessment of ventricular tachyarrhythmic events: a meta-analysis. Am Heart J 2012; 163 (3): 354-364.

8. Kracer B, Hintz SR, Van Meurs KP, Lee HC. Hypothermia therapy for neonatal hypoxic ischemic encephalopathy in the state of California. J. Pediatr 2014; 165: 267-273.

9. Gardiner J, Wagh D, McMichael J, Hakeem M, Rao S. Outcomes of hypoxic ischaemic encephalopathy treated with therapeutic hypothermia using cool gel packs: Experience from Western Australia. Eur J Paediatr Neurol 2014; 18: 391-398.

10. American College of Obstetricians and Gynecologists (ACOG). Neonatal encephalopathy and cerebral palsy: Executive summary. Obstet. Gynecol 2004; 103: 780-781.
11. Levene MI, de Vries L. Hypoxic-ischemic encephalopathy. 938-956. In: Martin RJ, Fanaroff AA, Walsh MC (Eds). Neonatal-Perinatal Medicine. Philadelphia: Elsevier, 2006.

12. Van Bel F, Walther FJ. Myocardial dysfunction and cerebral blood flow velocity following birth asphyxia. Acta Pediatr Scand 1990; 79: 756-762.

13. Alp H, Karaarslan S, Baysal T, Cimen D, Oran B. Normal values of left and right ventricular function measured by M-mode, pulsed Doppler and Doppler tissue imaging in healthy term neonates during a 1-year period. Early Human Develop 2012; 853-859.

14. Mori K, Nakagawa R, Nii M, Edagawa T, Takehara Y, Inoue M, Kuroda Y. Pulsed wave Doppler tissue echocardiography assessment of the long axis functions of the right and left ventricles during the early neonatal period. Heart Feb 2004; 90 (2): 175-180.

15. Wei Y, Xu J, Xu T, Fan J, Tao S. Left ventricular systolic function of newborns with asphyxia evaluated by tissue Doppler imaging. Pediatr Cardiol 2009; 30 (6): 741-746.

16. Nestaas E, Skranes JH, Stoylen A, Brunvand L, Fugelseth D. The myocardial function during and after whole-body therapeutic hypothermia for hypoxic-ischemic encephalopathy, a cohort study. Early Human Development 2014; 247-252.

17. Zhou H, Shao XM, Zhang XD, Chen C, Huang G. Effects of hypothermia on cardiac function in neonates with asphyxia. Zhongua Er Ke Za Zhi 2003; 41: 460-462.

18. Gebauer CM, Knuepfer M, Robel-Tillig E, Pulzer F, Vogtmann C. Hemodynamics among neonates with hypoxic-ischemic encephalopathy during whole-body hypothermia and passive rewarming. Pediatrics 2006; 117 (3): 843-850.

19. Sehgal A, Wong F, Mehta S. Reduced cardiac output and its correlation with coronary blood flow and troponin in asphyxiated infants treated with therapeutic hypothermia. Eur J Pediatr 2012; 171: 1511-1517.

20. Agrawal J, Shah GS, Poudel P, Baral N, Agrawal A, Mishra OP. Electrocardiographic and enzymatic correlations with outcome in neonates with hypoxic-ischemic encephalopathy. Ital J Pediatr 2012; 38: 33.

21. Costantini O, Hohnnloser SH, Kirk MM et al. The ABCD (Alternans Before Cardioverter Defibrillator) Trial: Strategies using T-wave alternans to improve efficiency of sudden cardiac death prevention. J Am Coll Cardiol 2009; 53: 471-479.

22. Van der Avoort CJ, Filion KB, Dendukuri N, Brophy JM. Microvolt T-wave alternans as a predictor of mortality and severe arrhythmias in patients with left-ventricular dysfunction: A systemic review and meta-analysis. BMC Cardiovascular Disord 2009; 9: 5-15.

23. Verrier RL, Klingenheben T, Malik $M$ et al. Microvolt T-Wave Alternans Physiological Basis, Methods of Measurement,and Clinical Utility: Consensus Guideline by International Society for Holter and Noninvasive Electrocardiology. J Am Coll Cardiol 2011; 58: 1309-1324.

24. Makarov L, Komoliatova V. Microvolt T-Wave Alternans during Holter Monitoring in Children and Adolescents. Ann Noninvasive Electrocardiol 2010; 15: 138-144.

25. Makarov L, Komoliatova V, Zevald S, Schmidt G, Muller A, Serebruanyd V. QT dynamicity, microvolt T-wave alternans, and heart rate variability during 24-hour ambulatory electrocardiogram monitoring in the healthy newborn of first to fourth day of life. J Electrocardiol 2010; 43: 8-14.

26. Tiainen M. Therapeutic Hypothermia after Cardiac Arrest; thesis, Department of Neurology, University of Helsinki, Helsinki, 2007.

Received June 26, 2017. Accepted July 17, 2017. 